\title{
Defining Double Negation Elimination
}

\author{
GREG-RESTAEL,- Department- of Phitosophty: Macquarie University,

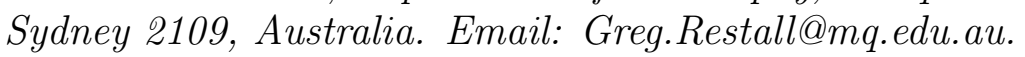 \\ Web: http://www.phil.mq.edu.au/staff/grestall/
}

\begin{abstract}
i:
Abstract

In his paper "Generalised Ortho Negation" [2] J. Michael Dunn mentions a cillaim of mine to the effect that there is no condition on 'perp frames' equivalent to the holding of double negation elimination $\sim \sim A \vdash A$. That claim is wrong. In this paper I correct my error and analyse the behaviour of conditions on frames for negations which verify a number of different theses. ${ }^{1}$
\end{abstract}

\section{Compatibility Frames}

in

Dunn's work on general models for negation has been a significant advance in our understanding of negation in non-classical logics $[1,2]$. These models generalise Kripke models for intuitionistic logic and Routley-Meyer models for relevant implication. I will recount the essential details of these models for negation here before we look at the behaviour of inference patterns such as double negation elimination.

Definition 1.1 $A$ frame is a triple $\langle P, C$, $\rangle$ consisting of a set $P$ of points, and two binary relations $C$ and $\sqsubseteq$ on $P$ such that

- $\sqsubseteq$ is a partial order on $P$. That is, $\sqsubseteq$ is reflexive, transitive and antisymmetric on $P$.

- $C$ is antitone in both places. That is, for any $x$ and $y$ in $P$, if $x C y, x^{\prime} \sqsubseteq x$ and $y^{\prime} \sqsubseteq y$ then $x^{\prime} C y^{\prime}$.

The relation $\sqsubseteq$ between points may be interpreted as one of information inclusion. A point $x$ is informationally included in $y$ if everything warranted by $x$ (or encoded by $x$ or made true by $x$ or included in $x$ or however else information is thought to be related to points) is also warranted by $y$. This is a transitive, reflexive relation on points. If the information content of a point is all you care about (as it is here) then antisymmetry is also an appropriate condition for inclusion.

$C$ is the relation of compatibility between points. ${ }^{2}$ A point $x$ is compatible with $y$ if and only if all claims excluded by $x$ are not warranted by $y$. It is clear that this merits the antitonicity condition - if $x$ is compatible with $y$, then $x^{\prime}$ (excluding no more than $x$ ) must be compatible with $y^{\prime}$ (warranting no more than $y$ ).

It is important to realise what "excluding" amounts to here. A point $x$ excludes a piece of information when it rules it out. A point might neither warrant nor exclude

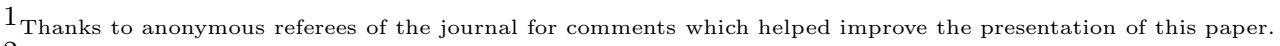

2 I use ' $C$ ' for a relation of compatibility instead of ' $\perp$ ' for the relation of incompatibility used by Dunn, simply because I use ' $\perp$ ' as the false propqsit $\overline{q q}_{\bar{n}} \overline{\mathrm{y}}_{\mathrm{y}} \mathrm{hich}$ entails all others. The difference is merely notational. $-11-{ }^{\prime}$ 
a particular piece of information, as it might be incomplete, and in certain peculiar circumstances it might both warrant and exclude the same piece of information, as it might be inconsistent. In this case, the compatibility relation $C$ will not be reflexive, as an inconsistent point is not compatible with itself - it warrants some information that it also rules out. If points are theory-like, then this is understandable. After all, a theory may well be inconsistent: it may assert something in one part which in another it might deny. Furthermore, compatibility in these models need not even be symmetric. The symmetry of compatibility is a substantive thesis which amounts to the inference from $A$ to $\sim \sim A$, as we shall see.

Given such a compatibility frame, we can interpret a language of conjunction, disjunction and negation in a straightforward way.

Definition 1.2 A relation $\vdash$ between points in $P$ and atomic propositions is an evaluation if it satisfies the hereditary condition:

- If $x \vdash p$ and $x \sqsubseteq y$ then $y \vdash p$.

The hereditary condition is an immediate consequence of intepreting $\sqsubseteq$ as informational inclusion. A frame with an evaluation relation is called a model, as we can model the entire language by extending the relation $\vdash$ to relate points to complex formulae.

Conjunction and disjunction, together with $\top$ and $\perp$ are interpreted as distributive lattice operators. For each point $x$ we require that:

- $x \vdash A \wedge B$ iff $x \vdash A$ and $x \vdash B$

- $x \vdash A \vee B$ iff $x \vdash A$ or $x \vdash B$

- $x \vdash \top$

- $x \forall \perp$

The condition for negation then utilises the compatibility relation:

- $x \vdash \sim A$ iff for each $y$ where $x C y, y \not \forall A$

This condition is warranted on the interpretation of compatibility. If $x$ is to be compatible with $y$ then if $x \vdash \sim A$ we cannot have $y \vdash A$. Conversely, if every $y$ where $x C y, y \forall A$, we ought have $x \vdash \sim A$, as $x$ has ruled $A$ out.

Example 1.3 Any intuitionistic frame $\langle P$, $\rangle$ is a compatibility frame, where we define $C$ by setting $x C y$ if and only if there is some $z$ where $x \sqsubseteq z$ and $y \sqsubseteq z$. Two points are compatible if and only if they have a common descendant. This definition of compatibility satisfies the antitonicity requirement.

This using this relation to define negation gives exactly the same answers as the traditional intuitionistic interpretation. If $\sim A$ is true at $x$ (according to the intuitionistic definition) and $x C y$, then $A$ must fail to be true at $y$, since $y$ and $x$ have a common descendant $z$, at which $A$ must fail. Conversely, if $A$ fails to be true at all points compatible with $x$, then $A$ must fail at all descendants of $x$, and so $\sim A$ is true at $x$ as desired.

Example 1.4 A frame for the negation fragment of relevant implication involves a set $P$ of points, an inclusion relation $\sqsubseteq$, and an involution * in $P$, satisfying order 


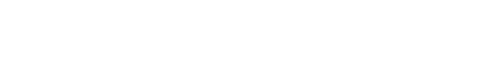

1. COMPATIBILITY FRAMES

inversion: if $x \sqsubseteq y$ then $y^{*} \sqsubseteq x^{*}$. Negation is defined using the ${ }^{*}$ operation: $x \vdash \sim A$ if and only if $x^{*} \forall A$.

We can define compatibility in terms of ${ }^{*}: x C y$ if and only if $x \sqsubseteq y^{*}$. Negation defined in terms of compatibility agrees with that defined by *, $a_{i}$ can be checked.

I have used Dunn's compatibility account of negation to give a philosophically respectable account of the semantics of negation in relevant logics [5]. The point here, however, is a formal one. I wish to consider how the addition of various conditions on the frame will modify the behaviour of the negation modelled by that frame. To do this we need to see how valid inference is modelled in frames.

Definition 1.5 An inference $A \vdash B$ is valid in the frame $F$ iff for each evaluation relation $\vdash$ on $F$, for each point $x$ if $x \vdash A$ then $x \vdash B$. Similarly, $A \vdash B$ is valid on the class $\mathcal{F}$ if and only if it is valid in each frame $F \in \mathcal{F}$.

To see how frames work, it is worthwhile to check some inferences.

Example 1.6 $\top \vdash \sim \perp$ is valid in every frame. Any point $x$ makes $T$ true. It suffices to show that any point also makes $\sim \perp$ true. But this holds trivially: for any point $y$ compatible with $x$, we have $y \forall \perp$ by the definition of $\perp$.

Example $1.7 \sim A \wedge \sim B \vdash \sim(A \vee B)$ is valid in every frame. If $x \vdash \sim A \wedge \sim B$, then for every point $y$ compatible with $x, y \forall A$ and $y \nvdash B$. Therefore $y \nvdash A \vee B$, and as $a$ result, $x \vdash \sim(A \vee B)$.

Example 1.8 If $A \vdash B$ is valid in a frame, so is $\sim B \vdash \sim A$. If $x \vdash \sim B$, then for each $y$ where $x C y$, we must have $y \forall B$. Since $A \vdash B$ we must have $y \forall A$, and as $a$ result, $x \vdash \sim A$ as desired.

Example 1.9 $A \vdash \sim \sim A$ is not valid on all frames. Consider the frame on two points $\{x, y\}$ in which $C$ is reflexive, and $x C y$ but not $y C x$. Let $x \vdash A$ but $y \forall A$. Then since $y \vdash \sim A$, we have $x \forall \sim \sim A$. The inference from $A$ to $\sim \sim A$ fails in this frame.

It is rather simple to spot the feature of this example responsible for the failure of $A \vdash \sim \sim A$. If $C$ were symmetric, then if $x \vdash A$ then we must have $x \vdash \sim \sim A$, for if $x C y$ since $y C x, y \forall \sim A$, and hence $x \vdash \sim \sim A$ as desired.

This correspondence between the validity of $A \vdash \sim \sim A$ and the symmetry of $C$ is two-way, as we can show that in any frame in which $C$ is not symmetric, we can invalidate $A \vdash \sim \sim A$. How? Select a failure of symmetry: some $x$ and $y$ where $x C y$ and not $y C x$. Define $\vdash$ by setting $z \vdash A$ if and only if $x \sqsubseteq z$. Now we will show that $x \nvdash \sim \sim A$, by showing that $y \vdash \sim A$. But this is straightforward: if $y C z$ and $z \vdash A$ then $x \sqsubseteq z$ would give us $y C x$ by the antitonicity of $C$. But this is not allowed, so $y \vdash \sim A$ and hence $x \nvdash \sim \sim A$ as desired. Thus, we have the following result:

Theorem 1.10 $A \vdash \sim \sim A$ is valid in a frame if and only if the compatibility relation on the frame is symmetric.

We say in this case that the symmetry of $C$ corresponds to the validity of $A \vdash \sim \sim A .^{3}$

3 This sense of 'correspondence' is well known in modal logics. For an overview of results in correspondence theory, consult van Benthem's survey article [6]. 
Another simple frame invalidates other well-known negation properties. Take a frame with one point $x$ which fails to be compatible with itself. Here $\sim \top \vdash \perp$ fails, as $x \vdash \sim \top$, and $A_{-1}^{\top} \sim A \vdash \perp$ fails as $x \vdash \top \wedge \sim \top$. These failures disappear in other restricted classes of frames, and it is trivial to expand our catalogue of correspondence results as follows [3].

Theorem $1.11 \sim \top \vdash \perp$ corresponds to $\forall x \exists y(x C y)$.

$A \wedge \sim A \vdash \perp$ corresponds to $\forall x(x C x)$.

We ought underline that correspondence results are not the same as soundness and completeness results. We may have a correspondence result without a completeness result if the canonical model for the logic fails to satisfy the correspondence condition. In this case the model (with its priveleged evaluation relation) will validate the theses of the logic, but the canonical frame underlying the model will not. In the other direction, it is possible to have conditions for which a logic is sound and complete, but to which the logic does not correspond. For example, S4 is sound and complete for finite tree models of modal logic, but the condition of being a finite tree does not correspond to the logic S4, for there are S4 frames which are not finite trees. We will see an example of this phenomenon in compatibility frames for negation in the next section.

\section{Double Negation Elimination}

Dunn asked the question: to what does double negation elimination, $\sim \sim A \vdash A$, correspond [2]? In this section we will examine how double negation elimination works in our frames.

To begin our investigation, note that $\sim \sim A \vdash A$ is independent of $A \vdash \sim \sim A$. We can construct a frame in which $\sim \sim A \vdash A$ is valid, but in which $A \vdash \sim \sim A$ fails as follows: Consider the three points $x, y, z$ such that $x \sqsubseteq y$, and such that $x C z, y C z$ and $z C x$. We can verify that double negation elimination holds. If $x \vdash \sim \sim A$ then $z \nvdash \sim A$ and hence $x \vdash A$. If $y \vdash \sim \sim A$ then $z \nvdash \sim A$ and hence $x \vdash A$, and $x \sqsubseteq y$ gives $y \vdash A$. If $z \vdash \sim \sim A$ then $x \nvdash \sim A$ gives $z \vdash A$ as desired. However, $C$ is not symmetric, so $A \vdash \sim \sim A$ fails in this frame. So, we have frames in which double negation elimination succeeds but double negation introduction fails. The converse is obvious: there are many models in which double negation introduction succeeds but elimination fails (take frames for intuitionistic logic as an example).

What does double negation elimination do to a logic? The first result is that it brings with it a de Morgan law absent in weaker logics.

Lemma 2.1 In any model in which $\sim \sim A \vdash A$ holds, so does $\sim(A \wedge B) \vdash \sim A \vee \sim B$.

Proof. Since $\sim A \vdash \sim A \vee \sim B$ gives $\sim(\sim A \vee \sim B) \vdash \sim \sim A$, elimination, gives $\sim(\sim A \vee$ $\sim B) \vdash A$. Similarly, we have $\sim(\sim A \vee \sim B) \vdash B$ and hence, $\sim(\sim A \vee \sim B) \vdash A \wedge B$, by lattice properties. By contraposition, $\sim(A \wedge B) \vdash \sim \sim(\sim A \vee \sim B)$ and one more elimination gives $\sim(A \wedge B) \vdash \sim A \vee \sim B$ as desired.

So, all frames in which double negation elimination holds are also frames in which $\sim(A \wedge B) \vdash(\sim A \vee \sim B)$ holds. It is not hard to give a condition which holds in all and only those frames in which this de Morgan law holds. 
Theorem $2.2 \sim(A \wedge B) \vdash(\sim A \vee \sim B)$ corresponds to the frame condition

$$
\left(\forall x, y_{1}, y_{2}\right)\left(x C y_{1} \wedge x C y_{2} \rightarrow(\exists z)\left(\left(y_{1} \sqsubseteq z\right) \wedge\left(y_{2} \sqsubseteq z\right) \wedge x C z\right)\right)
$$

In other words, if $x$ is compatitible with both $y_{1}$ and $y_{2}$, there is another point $z$, including both $y_{1}$ and $y_{2}$, with- which $x$ is also compatible. The verification of this correspondence is not difficult [3]:

Proof. Suppose that condition (2.1) holds, and that the rule fails. That is, there is some $x$ where $x \vdash \sim(A \wedge B)$, and $x \forall \sim A \vee \sim B$. Therefore $x \forall \sim A$ and $x \forall \sim B$, giving us a pair $y_{1}$ and $y_{2}$ of points where $x C y_{1}, y_{1} \vdash A, x C y_{2}$ and $y_{2} \vdash B$. Our frame condition gives us a $z$ where $x C z, y_{1} \sqsubseteq z$ and $y_{2} \sqsubseteq z$. Clearly $z \vdash A \wedge B$ and $x C z$ contradicts our assumption that $x \vdash \sim(A \wedge B)$. Therefore the de Morgan law must hold.

Conversely, if (2.1) fails we have $x, y_{1}$ and $y_{2}$ where $x C y_{1}$ and $x C y_{2}$ and there is no $z$ where $y_{1} \sqsubseteq z$ and $y_{2} \sqsubseteq z$. We construct a failure of the de Morgan law as follows. Define $\vdash$ by setting $w \vdash A$ iff $y_{1} \sqsubseteq w$, and $w \vdash B$ iff $y_{2} \sqsubseteq w$. Since $y_{1}$ and $y_{2}$ have no common $\sqsubseteq$-descendants, there is no point $z$ for which $z \vdash A \wedge B$. Therefore $x \vdash \sim(A \wedge B)$. However, since $y_{1} \vdash A$, we have $x \nvdash \sim A$, and since $y_{2} \vdash B$, we have $x \nvdash \sim B$, giving us $x \nvdash \sim A \vee \sim B$ and the de Morgan law fails, as we desired.

To extend this to a soundess and completeness proof we need to show that the canonical model for a logic containing our de Morgan law also validates the frame condition. We do this by showing that it satisfies a stronger condition.

Lemma 2.3 The canonical model for a logic containing $\sim(A \wedge B) \vdash \sim A \vee \sim B$ satisfies the condition

$$
(\forall x)(\exists y(-x-\dot{C} y) \rightarrow \exists z \forall y(x C y \leftrightarrow y \sqsubseteq z))
$$

Condition (2.2) entails condition (2.1) since if $x C y_{1}$ and $x C y_{2}$ we have $y_{1}, y_{2} \sqsubseteq z$. This is a genuinely stronger condition, for there are models for (2.1) in which (2.2) fails. Take a frame in which the points are the finite subsets of $\omega$, and define compatibility as having an empty intersection. So, $\{1,2\}$ is compatible with $\{3,4,5\}$ but not with $\{0,1\}$. The inclusion relation is $\subseteq$, and antitonicity holds can be verified. So $(2.1)$ is satisfied. If $x$ is compatible with both $y_{1}$ and $y_{2}$, then it is compatible with their union. However, there is no largest set compatible with $x$, for any $x$, so (2.2) fails.

However, the canonical model for a logic with the de Morgan law must, satisfy (2.2) as the following proof shows.

Proof. In the canonical model, the points are non-trivial prime theories [4]. In other words, they are sets of sentences closed under consequence (if $A \vdash B$ holds and $A \in X$ then $B \in X$ ) and conjunction (if $A, B \in X$ then $A \wedge B \in X$ ), respecting the constants ( $T \in X$ and $\perp \notin X$ ) and disjunction (if $A \vee B \in X$ then $A \in X$ or $B \in X$ ). The compatibility relation on this model is defined by setting $X C Y$ iff for each $\sim A \in X$, $A \notin Y$, and the subset relation $\subseteq$ does duty for the inclusion relation.

Suppose $\sim(A \wedge B) \vdash \sim A \vee \sim B$ holds in our logic. It suffices to show that for every non-trivial prime theory $X$ for which $X C Y$ for some $Y, X^{*}$ is a prime theory, when $X^{*}$ is defined as $\{A: \sim A \notin X\} . X C Y$ iff $Y \subseteq X^{*}$ by definition of $C$.

If $X C Y$ for some $Y$, then $\top \in Y$ ensures that $\sim \top \notin X$ and hence $\top \in X^{*}$. Since $\sim \perp \in X$ (as $\top \vdash \sim \perp$ is valid in any of our logics) it follows that $\perp \notin X^{*}$ as desired, 
so $X^{*}$ respects the constants. Now for consequence, if $A \vdash B$ is valid and $A \in X^{*}$ then $\sim A \notin X$, giving $\sim B \notin X$, and $B \in X^{*}$ as $\sim B \vdash \sim A$ must hold.

If $A, B \in X^{*}$ then $\sim A \notin X$ and $\sim B \notin X$, giving $\sim A \vee \sim B \notin X$ (by primeness of $X)$ and hence $\sim(A \wedge B) \notin X$, by our de Morgan law. This gives $A \wedge B \in X^{*}$ as desired.

Finally, for primeness, we have already seen that $\perp \notin X^{*}$ as $\sim \perp \in X$, and if $A \vee B \in X^{*}$ then $\sim(A \vee B)_{-} \notin X$ gives $\sim A \wedge \sim B \notin X$, and hence $\sim A \notin X$ or $\sim B \notin X$, i. e. $A \in X^{*}$ or $B \in X^{*}$ as desired.

So, $X^{*}$ is defined on condition that $X$ is compatible with something. So, the canonical model satisfies $(2.2)$, as we set out to prove.

That deals adequately with our de Morgan law. For double negation elimination itself, consider what we need in a frame in order to verify $\sim \sim A \vdash A$. We must have whenever $x \vdash \sim \sim A, x \vdash A$. Now $x \vdash \sim \sim A$ iff for every $y$ where $x C y, y \forall \sim A$. That is, there's some $z$ where $y C z$ where $z \vdash A$. This must hold wherever we take $A$ to be true. So, we need

$$
\forall X \forall x(\forall y(x C y \rightarrow(\exists z)(y C z \wedge z \in X)) \rightarrow x \in X)
$$

where $X$ ranges over all upwardly closed sets. This is not yet a proper frame condition, for it uses a second-order quantification.

The somewhat surprising result, crucial to this paper, is the fact that this is not essential. We will show that this condition is equivalent to a first order condition on frames.

Lemma 2.4 The frame condition (2.3) holds on a frame if and only if

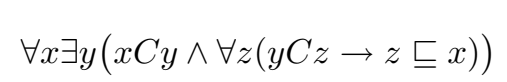

Proof. First, let us show that (2.4) follows from (2.3). Suppose that we have a point $x$. We wish to show that there is a $y$ where $x C y$ and $\forall z(y C z \rightarrow z \sqsubseteq x)$. Suppose, to the contrary, that there is no such $y$. It follows that for each $y$ where $x C y$ there is some $z$ where $y C z$ and $z \nsubseteq x$. For each $y$ we choose one such $z$, and call the result $f(y)$. It follows that if $x C y$ then $y C f(y)$ and $f(y) \nsubseteq x$. Now, consider the following set

$$
\quad X=\{w: \exists y(x C y \text { and } f(y) \sqsubseteq w)\}
$$

This set is closed upward under $\sqsubseteq$. As a result, it is an appropriate set to substitute for $X$ into (2.3). By construction, $x \notin X$. However, note that we do have $y(x C y \rightarrow$ $(\exists z)(y C z \wedge z \in X))$, since if $x C y$ then $f(y) \in X$ and $y C f(y)$. This then gipes $x \in X$, contradicting our construction. We have shown that (2.4) follows, given $(2: 3)^{4}$.

Now consider any set $X$ such that $\forall y(x C y \rightarrow(\exists z)(y C z \wedge z \in X))$. Giveng (2.4), we ' haglve a $y$ 'whlere $x C y$ and whenever $y C z, z \sqsubseteq x$. It follows that for some $z$ where $y C z$, $z \in X$, and thus, since $X$ is closed upwards, under $\sqsubseteq, x \in X$, confirming (2.3). Thus $(2.3)$ and $(2.4)$ are equivalent in any frame.

Condition (2.4) is a first-order condition, but it is not particularly perspicuous. It does not appear to tell us much about compatibility or negation in frames. However, it can be massaged into a nicer form. First, define the relation $I$ (of ' $x I y$ ' is ' $x$ is an inverse to $y^{\prime}$ ) as follows 
Then we have the follotring result:

$$
x I y \text { if and only if } \forall z(x C z \leftrightarrow z \sqsubseteq y)
$$

Lemma 2.5 Condition (2.4) holds in a frame if and only if

$$
\forall x \exists y(x I y \wedge \forall z(y I z \rightarrow z \sqsubseteq x))
$$

Condition (2.5) looks much more like a condition about double negation elimination. It asserts that in the frame each point has an 'inverse' which piles together all of the information compatible with that point, and furthermore, any inverse of an inverse of a point is contained 'in the point itself.

Proof. Given (2.5), (2.4) follows straightforwardly. Given any $x$, there is an $y$ where $x I y$. We have $x C y$ as $y \sqsubseteq y$. It suffices then to show that $\forall z(y C z \rightarrow z \sqsubseteq x)$, as we already have that $x C y$. But suppose $y C z$ : then since $y$ has an inverse (say, $y^{\prime}$ ) we know that $y^{\prime} \sqsubseteq x$; and we know too that since $y^{\prime}$ is $y^{\prime}$ 's inverse, $z \sqsubseteq y^{\prime}$. As a result, we have $z \sqsubseteq x$, as ${ }^{2}$ we wished to prove.

Conversely, given (2.4), it suffices to show that for each $x$ there inverse with the appropriate properties. We have for each $x$ some $y$ where $x C y^{\prime}$ anjd $\forall z(y C z \rightarrow z \sqsubseteq x)$. We will show that this $y$ is an inverse for $x$. For that we need just show that for all $v, x C v$ iff $v \sqsubseteq y$, as we already have $x C y$. Applying condition (2.4) to $y$ now, we see that there is a $w$ such that $y C w$ and $\forall z(w C z \rightarrow z \sqsubseteq y)$. Now since $y C w$ we have $w \sqsubseteq x$. Now take any $v$ where $x C v$. Since $y C w$, we have $w \sqsubseteq x$, and hence, $w C v$. But this means that $v \sqsubseteq y$. It follows that whenever $x C v, v \sqsubseteq y$. But since $x C y$, by antitonicity, $x C v$ if and only if $v \sqsubseteq y$ as desired. Therefore our choice of $y$ will suffice - in this frame. i. 1

In the canonical model for any logic including double negation elimination, condition (2.5) is verified, since firstly, $X^{*}$ is always a prime theory, as $\sim \perp \vdash \top$ gives $\sim \top \vdash \sim \sim \perp$, and by double negation elimination, $\sim_{-1}^{\top} \vdash \perp$, so $\sim \top \notin X$ and hence $\top \in X^{*}$. Secondly, $X^{*}$ acts as required since the appropriate de Morgan law holds in our logic, and we may apply the result of Lemma 2.3. Thirdly, if $A \in X^{* *}$ then $\sim A \notin X^{*}$, and hence $\sim \sim A \in X$, giving $A \in X$, so $X^{* *} \subseteq X$. So, we have also proved the following theorem.

$$
\text { : : : }
$$

Theorem 2.6 The logic of double negation elimination is sound and complete for the class of frames satisfying condition (2.5), and furthermore, double negation elimination corresponds to the holding of condition (2.5).

It remains only to explain why I claimed that there was no condition corresponding to double negation elimination. The reason is simple: I was confused by a weaker result. Double negation introduction, and the other simple rules have conditions which appeal only to the behaviour of the compatibility relation $C$. The conditions for double negation elimination and the de Morgan law we examined appeal essentially to inclusion. Here is why: Consider compatibility frames without inclusion. (This amounts to identifying $\sqsubseteq$ with identity.) Here, double negation elimination holds in a frame in only when

$$
\forall x \exists y(x C y \wedge \forall z(y C z \rightarrow x=z))
$$


In this case double negation introduction holds also, for this condition implies the symmetry of $C$. (You can reason as follows. Suppose $x C x^{\prime}$. By our condition, there is a $y$ where $x C y$, and $(\forall z)(y C z \rightarrow x=z)$. Now the condition applies equally well to $y$. So, there is a point $w$ where $y C w$, and $(\forall z)(w C z \rightarrow y=z)$. Now, since $y C w$, we have $w=x$ (and therefore $y C x$ ). And therefore, if $x C z$ we have $y=z$. It follows that $y=x^{\prime}$, and therefore $x^{\prime} C x$ as desired.)

However, we know that double negation introduction and elimination do not stand or fall together. In particular, in the canonical frame for the logic of double negation elimination, $\forall x \exists y(x C y \wedge \forall z(y C z \rightarrow x=z))$ fails. (There are theories $X$ for which $X \neq X^{* *}$.)

In this case, we have correspondence without completeness. Double negation elimination corresponds to a $C$-only frame condition, but this condition is too strong to allow a proof of completeness. To get a condition 'just right' we need the inclusion relation as well.

Double negation elimination can be modelled in compatibility frames in clear and natural way. But the most natural way requires us to talk about compatibility and inclusion - not compatibility alone.

\section{References}

[1] J. Michael Dunn. Star and perp: Two treatments of negation. In James E. Tomberlin, editor, Philosophical Perspectives, volume 7, pages 331-357. Ridgeview Publishing Company, Atascadero, California, 1994

[2] J. Michael Dunn. Generalised ortho negation. In Heinrich Wansing, editor, Negation: A Notion in Focus, pages 3-26. Walter de Gruyter, Berlin, 1996.

[3] Greg Restall. Combining possibilities and negations. Studia Logica, 59:121-140, 1997. Special Issue on Combining Logics.

[4] Greg Restall. An Introduction to Substructural Logics. Routledge, 2000.

[5] Greg Restall. Negation in Relevant Logics: How I stopped worrying and learned to love the Routley star. In Dov Gabbay and Heinrich Wansing, editors, What is Negation?, volume 13 of Applied Logic Series, pages 53-76. Kluwer Academic Publishers, 1999.

[6] Johan van Benthem. Correspondence theory. In Dov M. Gabbay and Franz Günthner, editors, Handbook of Philosophical Logic, volume 2, pages 167-247. Reidel, Dordrecht, 1984.

Received 22 September 1997, Revised 4 March 2000 